\title{
Optical Coherence Tomography, Scanning Laser Polarimetry and Confocal Scanning Laser Ophthalmoscopy in Retinal Nerve Fiber Layer Measurements of Glaucoma Patients
}

\author{
Farsad Fanihagh ${ }^{1}$, Stephan Kremmer ${ }^{*}{ }^{1,2}$, Gerasimos Anastassiou ${ }^{1,2}$, Maurice Schallenberg ${ }^{2,3}$, \\ Klaus-Peter Steuhl ${ }^{2}$ and Michael Selbach ${ }^{1,2}$
}

\author{
${ }^{I}$ Department of Ophthalmology, Evangelisches Krankenhaus Gelsenkirchen, Munckelstr. 27, 45879 Gelsenkirchen, \\ Germany \\ ${ }^{2}$ Department of Ophthalmology, University of Duisburg-Essen, Hufelandstr. 55, 45122 Essen, Germany \\ ${ }^{3}$ Eye Hospital, HELIOS Klinikum Wuppertal, Heusnerstrasse 40, 42283 Wuppertal, Germany
}

\begin{abstract}
Purpose: To determine the correlations and strength of association between different imaging systems in analyzing the retinal nerve fiber layer (RNFL) of glaucoma patients: optical coherence tomography (OCT), scanning laser polarimetry (SLP) and confocal scanning laser ophthalmoscopy (CSLO).

Materials and Methodology: 114 eyes of patients with moderate open angle glaucoma underwent spectral domain OCT (Topcon SD-OCT 2000 and Zeiss Cirrus HD-OCT), SLP (GDx VCC and GDx Pro) and CSLO (Heidelberg Retina Tomograph, HRT 3). Correlation coefficients were calculated between the structural parameters yielded by these examinations. The quantitative relationship between the measured RNFL thickness globally and for the four regions (superior, inferior, nasal, temporal) were evaluated with different regression models for all used imaging systems.

Results: The strongest correlation of RNFL measurements was found between devices using the same technology like GDx VCC and GDx Pro as well as Topcon OCT and Cirrus OCT. In glaucoma patients, the strongest associations $\left(\mathrm{R}^{2}\right)$ were found between RNFL measurements of the two optical coherence tomography devices Topcon OCT and Cirrus OCT $\left(\mathrm{R}^{2}=0.513\right)$ and between GDx VCC and GDx Pro $\left(\mathrm{R}^{2}=0.451\right)$. The results of the OCTs and GDX Pro also had a strong quantitative relationship (Topcon OCT $\mathrm{R}^{2}=0.339$ and Cirrus OCT $\mathrm{R}^{2}=0.347$ ).

GDx VCC and the OCTs showed a mild to moderate association (Topcon OCT $\mathrm{R}^{2}=0.207$ and Cirrus OCT $\mathrm{R}^{2}=0.258$ ). The confocal scanning laser ophthalmoscopy (HRT 3) had the lowest association to all other devices (Topcon OCT $\mathrm{R}^{2}=$ 0.254 , Cirrus OCT $\mathrm{R}^{2}=0.158$, GDx Pro $\mathrm{R}^{2}=0.086$ and GDx VCC $\mathrm{R}^{2}=0.1$ ).

Conclusion: The measurements of the RNFL in glaucoma patients reveal a high correlation of OCT and GDx devices because OCTs can measure all major retinal layers and SLP can detect nerve fibers allowing a comparison between the results of this devices. However, CSLO by means of HRT topography can only measure height values of the retinal surface but it cannot distinguish between different retinal layers.

This may explain the rather poor correlations and associations between CSLO measurements and those of all other imaging devices which makes it difficult to compare HRT 3 nerve fiber data.

These correlations are important in clinical routine especially when different techniques are used in the follow-up of glaucoma patients.
\end{abstract}

Keywords: Confocal scanning laser ophthalmoscopy, glaucoma, OCT, retinal nerve fiber layer, scanning laser polarimetry.

\section{INTRODUCTION}

The term glaucoma describes a couple of diseases that have one feature in common: the retinal ganglion cells and their axons are severely damaged. Untreated, apoptosis causes ganglion cell death which subsequently leads to typical glaucomatous damage at the optic nerve head, defects

*Address correspondence to this author at the Department of Ophthalmology, Evangelisches Krankenhaus Gelsenkirchen, Munckelstr. 27, 45879 Gelsenkirchen, Germany; Tel: 0049 - 209 - 37261;

Fax: 0049 - 209 -378555; E-mail: stkrem@gmail.com of the visual fields and - if not or unsuccessfully treated ultimately to blindness. It is well known that patients with glaucoma can suffer a $20 \%$ to $50 \%$ loss of retinal ganglion cells before a defect becomes evident in standard perimetry $[1,2]$. In a couple of studies, RNFL defects were discovered in more than $50 \%$ of eyes with glaucoma approximately 5-6 years before functional deficits became manifest in these patients' visual fields [3, 4]. To prevent glaucomatous damage, it is important to detect changes of the retinal ganglion cells and their nerve fiber layers as early as possible and to monitor their follow-up closely in order to find an adequate treatment of glaucoma and to control its efficiency. 
The classical way of documenting morphological alterations, ophthalmoscopy and photography of the optic disc and the retinal nerve fiber layer (RNFL), suffer from interobserver variability, technical problems e.g. in cases of cataract and narrow pupils, and often do not detect diffuse atrophy of the RNFL [5] which is an early sign of glaucomatous damage of the retinal ganglion cells and their axons.

Fortunately, modern imaging techniques have provided the ophthalmologist with the means to detect early manifestations of glaucomatous damage as well as to track changes that could result in visual impairment if therapy is not adjusted accordingly. These techniques are optical coherence tomography (OCT), scanning laser polarimetry (SLP) and confocal scanning laser ophthalmosopy (CSLO). Each of these diagnostic tools is based on different imaging modalities.

Optical coherence tomography applies the principle of interferometry to determine the interface between different ocular tissues. With automated segmentation algorithms based on reflectivity changes between the different layers of the retina, retinal nerve fiber layer thickness can be calculated. Time-domain OCT (TD OCT) has for a couple of years been used as an imaging technology for a variety of posterior segment diseases, besides glaucoma particularly for the diagnosis and staging of macular pathologies like agerelated macular degeneration (AMD) and macular edema. Spectral domain OCT (SD OCT) is a more advanced application of this technology, allowing much faster scanning and higher resolution images than conventional TD OCT.

SLP is based on the principle of retardance of backscattered light through the birefrigent RNFL which translates into, and thus allows, a precise measurement of this layer's thickness. The most recent versions of this technology are outfitted with variable corneal compensation (VCC, GDx VCC) and enhanced corneal compensation (ECC, GDx Pro).

Confocal scanning laser tomography, CSLO (Heidelberg Retina Tomograph, HRT 3) projects a laser grid across the retina and records multiple layers of focal depth. The technology generates a three-dimensional image of the surface of the optic disc and surrounding tissues.

With regards to the importance of the RNFL in the early detection of glaucoma as well as of disease progression, precise and reproducible measurements of this anatomical structure are important. Therefore, many patients undergo one or more of these new diagnostic imaging procedures depending on the preference of their ophthalmologist or availability of the expensive devices. But during their lifetime patients can move, the ophthalmologist may change or new imaging technologies like e.g. FD OCT come up and during the follow-up of a patient different devices may be used.

Therefore, the knowledge of the extent to which these methods correlate is important for many treatment decisions.

The aim of our study was to compare the RNFL parameters obtained by two different OCT devices, Topcon SD-OCT 2000 and Cirrus HD-OCT, by two different SLP devices, GDx VCC and GDx Pro, as well as CSLO by HRT
3 in glaucoma patients, to evaluate if they correlate and to find a quantitative functional relationsship between them.

\section{MATERIALS AND METHODOLOGY}

All patients enrolled in this study attended the Department of Ophthalmology, Evangelisches Krankenhaus Gelsenkirchen, Germany because of moderate open angle glaucoma, with both, typical morphological changes of the optic disk according to Jonas [6] and related glaucomatous visual field defects according to Aulhorn [7].

A total of 114 eyes of 114 Caucasian subjects were included in the study. One eye of each patient was randomly selected if both eyes were eligible.

As a basic clinical evaluation, all patients underwent a standard ophthalmic examination including tests for the bestcorrected visual acuity, slit-lamp inspection, Goldmann applanation tonometry, pachymetry, gonioscopy, funduscopy, white on white perimetry with Oculus Twinfield II and optic disc evaluation.

Exclusion criterias were ocular surgery with the exception of uncomplicated cataract surgery, refractive errors outside $+/-3$ diopters, and any ocular abnormality other than glaucoma.

After these basic examinations, all patients underwent the following five imaging techniques:

- $\quad$ spectral domain optical coherence tomography with the Cirrus HD-OCT (Carl Zeiss Meditec, Dublin, California) and the Topcon SD-OCT 2000 (Topcon Corporation, Tokyo, Japan),

- $\quad$ scanning laser polarimetry (SLP) with GDx VCC as well as GDx Pro (Carl Zeiss Meditec, Dublin, California),

- confocal scanning laser ophthalmoscopy (CSLO) with Heidelberg Retina Tomograph (HRT 3, Heidelberg Engineering, Heidelberg, Germany).

The Cirrus HD-OCT provides RNFL data obtained at a circle line with a diameter of $3.46 \mathrm{~mm}$ centered at the optic disc. The parameters are the average RNFL thickness globally, RNFL symmetry, the RNFL values separately divided into quadrants and divided in clock hours. The standard Topcon SD-OCT parameters which were used in our study are similar to the Zeiss Cirrus HD-OCT but the instrument allows an easy individual adjustment with regards to e.g. small or large optic disks. The GDx VCC and GDx Pro measured the RNFL thickness in a circle with diameters between $2.6 \mathrm{~mm}$ and $3.2 \mathrm{~mm}$ centered at the optic disk. Their printouts reveal data of the RNFL thickness with standard deviation, RNFL symmetry, superior and inferior RNFL thickness and Nerve fiber indicator (NFI). The HRT 3 measures RNFL thickness data directly at the rim of the optic disk and it is divided in quadrants.

Statistical analyses were performed with JMP version 10 (SAS Institute Inc., Cary, NC) and SIGMA Plot version 11.0 (Systat Software Inc., San Jose, CA). Results were expressed as mean \pm standard deviation. The normal distribution was evaluated with the Kolmogorov-Smirnov and the chi square test for all parameters. All global, superior and inferior nerve fiber layer parameters were normally distributed. The 
Pearson correlation was used to assess the linear relationship between these device parameters. The normal distribution in temporal and nasal parameters was not allways given. Parameters of these sectors were proven by the Spearman's rank correlation coefficient. Linear and non-linear regression analyses were used to compare and to study the relationship between RNFL measurements of the different devices. Since we used data of the same patients, we have expected a strong linear relationship. The linear model $\left(\mathrm{y}=\mathrm{a}+\mathrm{b}^{*} \mathrm{x}\right)$ was compared with non-linear models, logarithmic $(y=b * \ln (x)+a)$, potential $\left(y=a^{*} x^{\wedge} b\right)$, hyperbolic $1(y=a+b / x)$, hyperbolic $2\left(y=1 /\left(a+b^{*} x\right)\right.$ and hyperbolic $3\left(\mathrm{y}=\mathrm{x} /\left(\mathrm{a}+\mathrm{b}^{*} \mathrm{x}\right)\right.$ regression analysis were determined between RNFL thickness globally and for the four regions (superior, inferior, nasal, temporal). The goodness-of-fit of any particular regression model is expressed by the coefficient of determination $\left(\mathrm{R}^{2}\right)$. The same analysis was used for superior and inferior average RNFL thickness values. The TSNIT graph compares RNFL thickness data from a circle around the optic disc in the order temporal, superior, nasal, inferior, temporal (TSNIT) to the normative database.

\section{RESULTS}

Nerve fiber layer measurements of 114 eyes of 114 patients with open angle glaucoma were enrolled in the present study. 38 patients were male and 76 female. The age ranged from 20 to 83 years (mean age 67.5 years).

The univariate statistics of the nerve fiber analysis shows the differences in RNFL parameter values between the different devices. As expected, GDx VCC and GDx Pro have mean values in the same range and the average results of the OCT devices are higher. The scale of the RNFL mean value of HRT 3 differs significantly (Fig. 1).

Despite the differences in range, RNFL profiles are statistically comparable.

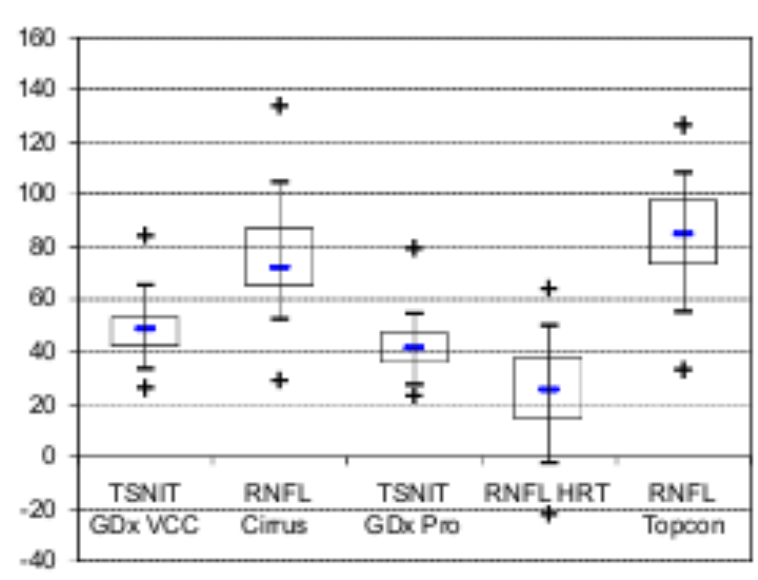

Fig. (1). Box-and-wisker graphs showing nerve fiber layer thickness measurements of the different devices in glaucoma patients. Results of HRT 3 and GDx measurements were lower than OCT values.

In glaucoma patients correlations were strongest between Topcon OCT 2000 and Cirrus HD-OCT $(0.716, p<0.0001)$ as well as between GDx Pro and GDx VCC $(0.672$, p < 0.0001 ) for the global nerve fiber layer measurements.
RNFL parameters assessed by optic coherence tomography (Topcon OCT 2000 and Cirrus HD-OCT) showed strong correlations to GDx Pro (Topcon 0.582, p < 0.0001; Cirrus $0.589, \mathrm{p}<0.0001)$ and moderate correlations to GDx VCC (Topcon 0.455, p <0.0001; Cirrus 0.508, $\mathrm{p}$ $<0.0001)$ data. HRT 3 shows a moderate correlation to the Topcon OCT $(0.504, \mathrm{p}<0.0001)$. Mild or no significant correlations were found between HRT 3 and the results of the other devices (GDx VCC 0.317, p <0.0001; GDx Pro $0.293, p<0.0001$; Cirrus 0.397, $p<0.0001$ ). The four regions of the optic nerve were analyzed separately, normal distributed data (superior, inferior) with the Pearson correlation and the others with the Spearman's rank correlation. The superior and inferior data show the same behavior as the global nerve fiber data. The temporal and nasal measurements reveal a lower correlation and differ from the global data.

As could be expected by the global data, the correlation between the parameters (superior, inferior) of the GDx VCC and GDx Pro devices was very high (superior 0.714, $\mathrm{p}$ $<0.0001$; inferior $0.755, \mathrm{p}<0.0001$ ). There was a high correlation calculated between the areas of the Topcon SDOCT and Cirrus HD-OCT (superior 0.670, p <0.0001; inferior $0.695, \mathrm{p}<0.0001$ ). Furthermore, there was a moderate or strong correlation between the Topcon SD-OCT and Cirrus HD-OCT and the GDx devices which was determined between 0.45 and $0.57(\mathrm{p}<0.0001)$. Only the HRT 3 showed a lower correlation to the other devices $(<0.4$, $\mathrm{p}<0.0001$ ) (Fig. 2).

After finding strong correlations between the devices the structure-function association of linear and non-linear functions were determined. The goal was to find that the association is strong enough by linear regression function. GDx Pro has a high coefficient of determination $\left(\mathrm{R}^{2}\right)$ with GDx VCC in the linear regression analysis $\left(\mathrm{Y}=14.01+0.574 * \mathrm{X}, \mathrm{R}^{2}=0.451\right)$. The linear association was strong between Cirrus OCT and Topcon OCT $\left(\mathrm{Y}=14.82+0.71 * \mathrm{X}, \mathrm{R}^{2}=0.513\right)$. GDx Pro has a moderate linear association with both OCT devices (Topcon OCT: $\mathrm{Y}=37.82+1.11 * \mathrm{X}, \quad \mathrm{R}^{2}=0.339, \quad$ Cirrus OCT: $\mathrm{Y}=28.09+1.13 * \mathrm{X}, \mathrm{R}^{2}=0.347$ ). As was seen in the functional relationship analysis between HRT 3 and the GDx devices, the best match is in a linear function with a weak $\mathrm{R}^{2}$ (GDx Pro: $\mathrm{Y}=37.96+15.77^{*} \mathrm{X}, \mathrm{R}^{2}=0.086$ and GDx VCC: $\mathrm{Y}=$ $\left.43.61+19.9 * \mathrm{X}, \mathrm{R}^{2}=0.1\right)$.

The associations $\left(\mathrm{R}^{2}\right)$ between global and regional RNFL measurements of HRT 3 and OCT were also mild. The linear function was the best association between the HRT 3 and the OCTs (Topcon OCT: $\mathrm{Y}=71.56+51.90 * \mathrm{X}, \mathrm{R}^{2}=0.254$, Cirrus OCT: $\left.\mathrm{Y}=65.22+41.08 * \mathrm{X}, \mathrm{R}^{2}=0.158\right)$. GDx VCC also shows a weak association with the OCT devices (Topcon OCT: $\mathrm{Y}=48.38+0.745 * \mathrm{X}, \quad \mathrm{R}^{2}=0.207$, Cirrus OCT: $\mathrm{Y}=35.01+0.833 * \mathrm{x}, \mathrm{R}^{2}=0.258$ ).

The $\mathrm{R}^{2}$ values are calculated for each fit (Table 1). The linear associations were not clearly stronger between the different technologies as the non-linear functions.

Fig. (3) depicts the linear relationship between the devices. A linear fit could only be observed for the plots $\mathrm{c}, \mathrm{d}$, $\mathrm{i}, \mathrm{j}$. 


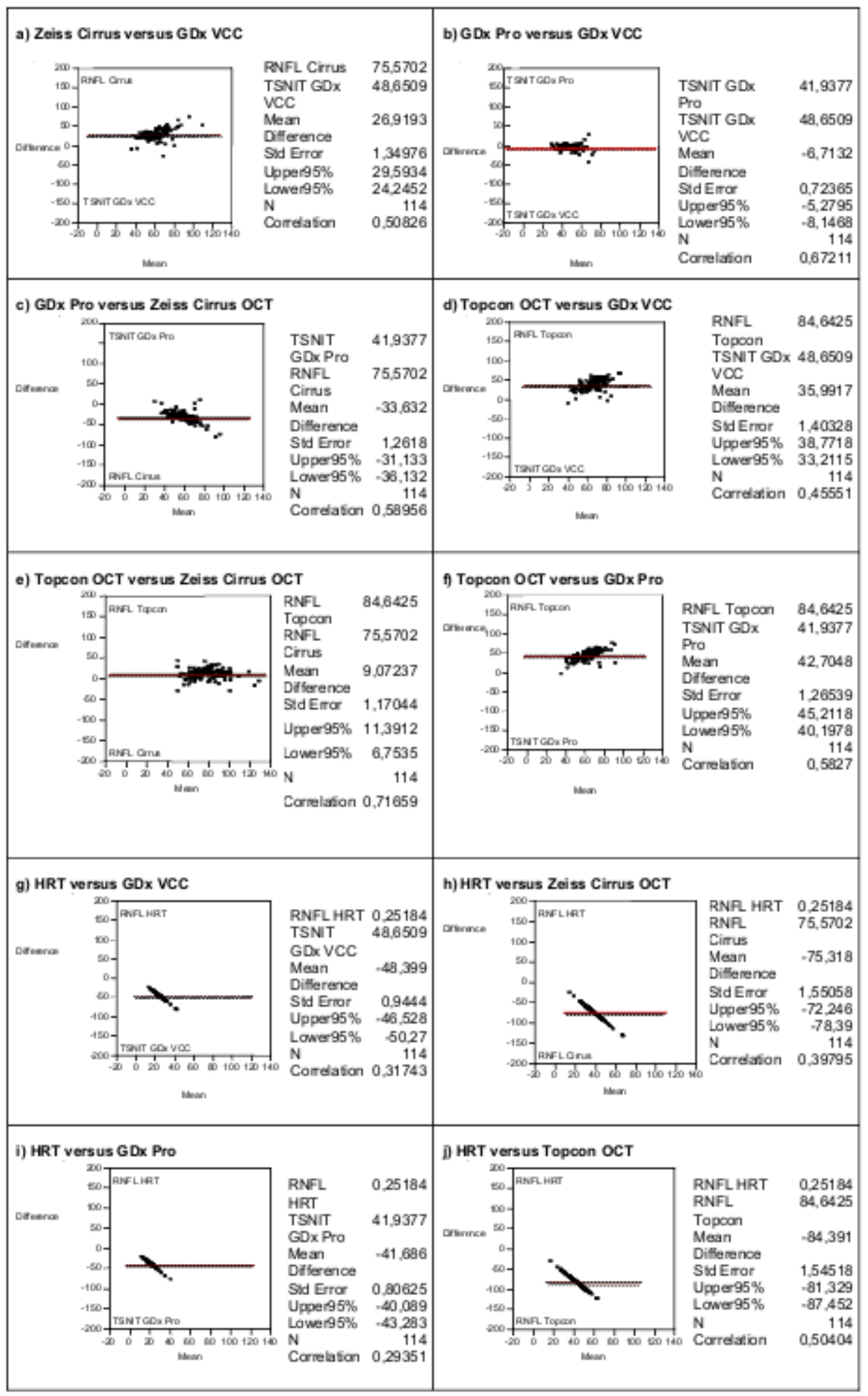

Fig. (2). Correlation of nerve fiber measurements obtained by the different devices. Between the OCTs (e), the GDx Pro and the OCTs (c, f), as well as GDx Pro and GDx VCC (b) a strong correlation can be observed. GDx VCC had a moderate correlation to the OCTs (a, d), as well HRT 3 and Topcon SD (j). HRT 3 revealed only mild or no significant correlation to the other devices $(\mathbf{g}, \mathbf{h}, \mathbf{i})$. 
Table 1. Data are shown for structure-function linear and non-linear associations $\left(R^{2}\right)$ between the RNFL measurements by the OCTs (Cirrus HD, Topcon FD), GDx Pro, GDx VCC and HRT 3. The associations between the OCTs, GDx Pro and the OCTs, GDx Pro and GDx VCC are significantly stronger than HRT 3 and GDx VCC to the other devices. Only the linear fits between the OCTs (Cirrus HD, Topcon SD) are significantly stronger as the non-linear fits (Two tailed paired t-test, $\mathbf{p}<\mathbf{0 . 0 0 0 1 )}$.

\begin{tabular}{|c|c|c|c|c|c|c|c|}
\hline Device 1 & Device 2 & $\begin{array}{c}\text { Linear } \\
\mathbf{R}^{2}, \mathbf{p}<0.0001\end{array}$ & $\begin{array}{l}\text { Logarithmic } \\
\mathbf{R}^{2}, \mathbf{p}<0.0001\end{array}$ & $\begin{array}{c}\text { Potential } \\
\mathbf{R}^{2}, \mathbf{p}<0.0001\end{array}$ & $\begin{array}{c}\text { Hyperbolic } \\
1 R^{2}, \mathbf{p}<0.0001\end{array}$ & $\begin{array}{c}\text { Hyperbolic } \\
2 \mathrm{R}^{2}, \mathbf{p}<0.0001\end{array}$ & $\begin{array}{c}\text { Hyperbolic } \\
3 \mathbf{R}^{2}, \mathbf{p}<0.0001\end{array}$ \\
\hline GDx VCC & GDx Pro & $\underline{0.451}$ & 0.485 & 0.521 & 0.485 & 0.456 & 0.553 \\
\hline GDx VCC & Topcon SD-OCT & 0.207 & 0.236 & 0.220 & 0.247 & 0.158 & 0.191 \\
\hline GDx VCC & HRT 3 & 0.1 & 0.118 & 0.001 & 0.127 & 0.026 & 0.030 \\
\hline GDx Pro & Topcon SD-OCT & $\underline{0.339}$ & 0.372 & 0.342 & 0.376 & 0.249 & 0.284 \\
\hline GDx Pro & HRT 3 & 0.086 & 0.332 & 0.001 & 0.355 & 0.123 & 0.150 \\
\hline Cirrus HD-OCT & Topcon SD-OCT & $\underline{0.513}$ & 0.483 & 0.421 & 0.384 & 0.334 & 0.266 \\
\hline Cirrus HD-OCT & HRT 3 & 0.158 & 0.129 & 0.146 & 0.028 & 0.136 & 0.036 \\
\hline
\end{tabular}

\section{DISCUSSION}

To the best of our knowledge, this is the first study evaluating the correlation between OCT, SLP, and CSLO in analyzing the RNFL in patients with open angle glaucoma. Our measurements with five of these modern imaging devices show a high correlation and association between OCT and SLP but not between either or both of them and confocal scanning laser ophthalmoscopy. This is also the case for the regression analysis of the association between the devices. The strongest quantitive linear relationships were found between Cirrus HD-OCT and Topcon SD-OCT 2000 data as well as between GDx Pro and GDx VCC data.

Recently, we were able to demonstrate that an undifferentiated comparison of RNFL thickness assessment between GDx VCC, GDx Pro and Spectralis OCT is not feasible. The measurements with SD OCTs are generally higher than with GDx VCC and GDx Pro, the reasons being physical differences of the imaging methods [8] but also software dependent. In the standard clinical glaucoma RNFL protocols and printouts of almost all OCT devices, blood vessels are not extracted from RNFL measurements [9]. This is crucial especially if glaucoma is progressing because nerve fibers are deceasing, RNFL is decreasing, and the ratio between RNFL and blood vessels is markedly changing. Although OCT technology can differentiate between RNFL and blood vessels (e.g. 2 mouse clicks in Topcon SD-OCT 2000) this helpful feature is not included in standard OCT RNFL software protocols. This is an advantage of SLP because blood vessels have no birefringent properties and therefore they do not disturb GDx RNFL measurements [9].

Nevertheless, we have documented in the present study that there is a reasonable degree of correlation between OCT and SLP but a much lesser correlation between these technologies and CSLO like the HRT 3. While highly valued for the examination of the optic nerve head and its excavtion, CSLO seems to be less suitable for analyzing the RNFL.
These findings are in accordance with other studies that have compared these technologies, usually more one-on-one than all three together as we have done [10-12].

In a recent study, SD OCT proved its worth in detecting preperimetric glaucoma. In a cohort of 134 eyes of 88 glaucoma patients, the parameter with the largest area under the receiver operating characteristic curves (AUC) was the temporal superior RNFL thickness $(0.88+/-0.03)$, followed by global RNFL thickness $(0.86+/-0.03)$ and temporal inferior RNFL thickness $(0.81+/-0.04)$. For CSLO performed with a HRT 3 , by contrast, the parameter with the largest AUC was the rim area $(0.72+/-0.05)$ followed by rim volume $(0.71+/-0.05)$ and linear cup-to-disc ratio $(0.66$ $+/-0.05)$. The authors conclude that SD OCT had a better performance as the CSLO assessing the RNFL in a cohort of glaucoma patients, some of them with no or little evidence of glaucomatous damage in fundus photography [10]. Comparing SD OCT and CSLO in eyes with glaucomatous visual field defects, Leung et al. [11] found an even higher AUC (0.978) for the SD OCT parameter global RNFL thickness. In that study, Cirrus HD-OCT demonstrated lower measurement variability compared with Stratus TD-OCT. The generally lower variability of the imaging instruments compared to previous studies was at least in part attributed to the less severe stage of glaucoma in their cohort - unlike our patients who suffered from moderate glaucoma damage. The same study group could demonstrate that the Cirrus HDOCT could detect changes in RNFL thickness sooner than the Stratus TD-OCT [13]. The performance of SD OCT in detecting RNFL defects in preperimetric glaucoma can further be enhanced by using a speckle noise-reduced version of that device [4]. Relying on imaging alone when measuring the RNFL, one should be aware of certain natural variations: some authors report that people with European descent have thinner average RNFL measurements than those with African and Chinese descent [14] but others show that RNFL of Europeans is thicker than that of Africans [15]. 


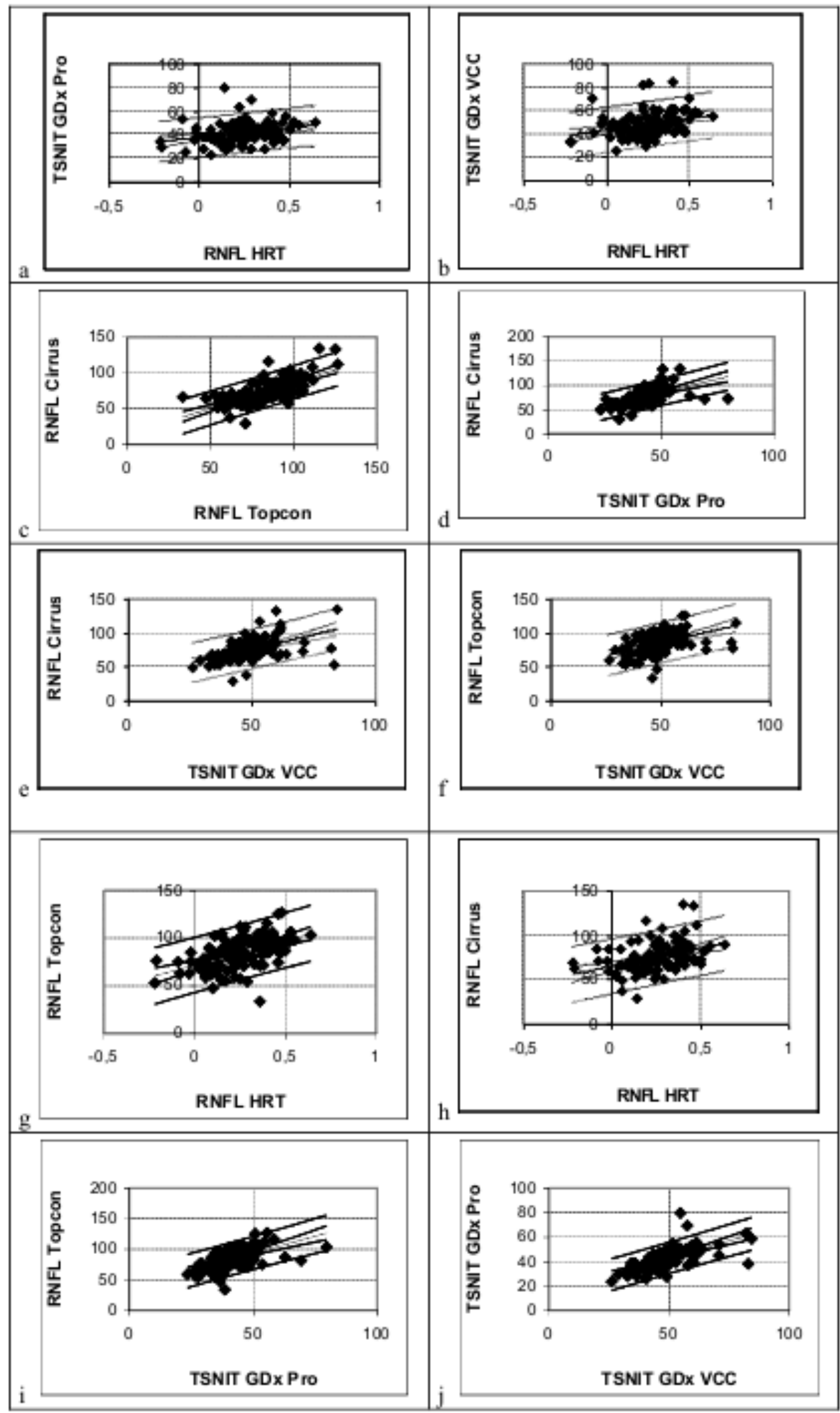

Fig. (3). Scatterplots showing the linear associations between the nerve fiber measurements from the OCTs (Cirrus HD, Topcon SD), GDx Pro, GDx VCC and HRT 3. The $\mathrm{R}^{2}$ values are calculated for each fit (Table 1). A linear fit was only significant for the plots $\mathbf{c}, \mathbf{d}$, $\mathbf{i}, \mathbf{j}$ $(\mathrm{p}<0.0001)$. 
An increasing RNFL thickness seems to be associated - for reasons not yet known - with a larger optic disc area [16]. A thinner average RNFL was observed in eyes with myopia and hyperopia [17]. The peculiarities of the RFNL bundles in myopic eyes (average spherical error: -5.44 diopters) have been described by Leung et al. [18]. Age was associated with several SD OCT parameters, with rim area reducing by $0.005 \mathrm{~mm}^{2} /$ year, RNFL thickness reducing by $0.18 \mu \mathrm{m} /$ year, and inner retinal thickness reducing by $0.1 \mu \mathrm{m} /$ year [15]. A Canadian group has demonstrated that measurements with the Stratus TD-OCT revealed a much higher variability than GDx Pro and GDx VCC devices [19].

Zhu H. et al. [20] could prove that data from the older TD OCTs have a lower reproducibility because measurements in the time domain are relatively slow and therefore data is only obtained for the scanning circle. New SD OCT techniques are much faster, record a plenty of data, and enable more stabile measurements.

When imaging 110 eyes of glaucoma patients and healthy controls in 6-months intervals for 1.5 to 3 years with a SD OCT and both a GDx VCC and a GDx Pro, there was significant higher variability with both SLP devices than with the RTVue SD-OCT.

The atypical retardation pattern (ARP) has a major impact on the RNFL thickness results of GDx devices and it is significantly lower in GDx Pro than in GDx VCC. This must be taken into consideration when evaluting RNFL thickness measurements and may be an explanation for the higher correlations of GDx Pro with SD OCT data [8].

The long-term measurement variability was higher in perimetric glaucoma than in healthy eyes with both GDx methods; no similar between-group differences were seen with the RTVue SD-OCT. The authors suggested that a parameter reflecting long-term polarimetric variability - as indicated by the higher variability of both GDx devices in perimetric glaucoma patients - might potentially be used to identify eyes with increased risk of future progression [21].

Our group has recently compared CSLO and SLP at different distances from the rim of the optic nerve head. The data gathered did not correlate very well, particularly the closer to the optic disc measurements have been taken. The strongest correlations could be found at a distance of 1.25 to 1.75 optic nerve head diameters from the papilla [22]. Unfortunately, the HRT 3 is the only device measuring RNFL values directly at the rim of the optic disc.

\section{CONCLUSION}

Whereas OCT can assess all major retinal layers and SLP can detect especially the RNFL, CSLO measures height values of the retinal surface and provides information of the sum of all tissues under the surface, but it cannot distinguish e.g. between RNFL, blood vessels and glial tissue. Additionally, the HRT is the only device measuring directly at the rim of the optic disc and it is dependent on an operator drawing a contour line and a 'reference plane' which is prone to intra- and interindividual variations, changes with progression of glaucoma, and is thus difficult to define.

This may explain the low correlations and quantitative relationships between CSLO measurements and those of all other imaging devices making it difficult to compare HRT 3 nerve fiber data.

These findings are important for the clinical management and therapy of glaucoma patients especially when different techniques are used in the follow-up.

\section{CONFLICT OF INTEREST}

The authors confirm that this article content has no conflict of interest.

\section{ACKNOWLEDGEMENTS}

Supported by Alfried Krupp von Bohlen und HalbachStiftung, Hügel 15, 45133 Essen, Germany.

\section{REFERENCES}

[1] Quigley HA, Addicks EM, Green WR. Optic nerve damage in human glaucoma. III. Quantitative correlation of nerve fiber loss and visual field defect in glaucoma, ischemic neuropathy, papilledema and toxic neuropathy. Arch Ophtalmol 1982; 100: 135-46.

[2] Kerrigan-Baumrind LA, Quigley HA, Pease ME, et al. Number of ganglion cells in glaucoma eyes compared with threshold visual field tests in the same persons. Invest Ophthalmol Vis Sci 2000; 41: 741-8.

[3] Sommer A, Katz J, Quigley HA, et al. Clinically detectable nerve fiber atrophy precedes the onset of glaucomatous field loss. Arch Ophthalmol 1991; 109: 77-83.

[4] Nukada M, Hangai M, Mori S, et al. Imaging of localized retinal nerve fiber layer defects in preperimetric glaucoma using spectraldomain optical coherence tomography. J Glaucoma 2014; 23: 1509.

[5] Mwanza JC, Durbin MK, Budenz DL, et al. Glaucoma diagnostic accuracy of ganglion cell-inner plexiform layer thickness: comparison with nerve fiber layer and optic nerve head. Ophthalmology 2012; 119: 1151-8.

[6] Jonas JB, Budde WM, Panda-Jonas S. Ophthalmoscopic evaluation of the optic nerve head. Surv Ophthalmol 1999; 43: 293-320.

[7] Aulhorn E, Karmeyer H. Frequency distribution in early glaucomatous visual field defects. Doc Ophthalmol 1977; 14: 7583

[8] Schallenberg M, Dekowski D, Kremmer S, et al. Comparison of Spectralis-OCT, GDxVCC and GDxECC in assessing retinal nerve fiber layer (RNFL) in glaucomatous patients. Graefe Arch Clin Exp Ophthalmol 2013; 251: 1343-53.

[9] Kremmer S. Moderne Nervenfaserdiagnostik - Fallbeispiele. In: Dietlein T, Krieglstein GK, Wiedemann P, Eds. Glaukom und Makula. Heidelberg: Springer Medizin Verlag 2011; 15-58.

[10] Leung CK, Cheung CY, Weinreb RN, et al. Retinal nerve fiber layer imaging with spectral-domain optical coherence tomography: a variability and diagnostic performance study. Ophthalmology 2010; 117: 267-74.

[11] Lisboa R, Leite MT, Zangwill LM, et al. Diagnosing preperimetric glaucoma with spectral domain optical coherence tomography. Ophthalmology 2012; 119: 2261-9.

[12] Medeiros FA, Vizzeri G, Zangwill LM, et al. Comparison of retinal nerve fiber layer and optic disc imaging for diagnosing glaucoma in patients suspected of having the disease. Ophthalmology 2008; 115: 1340-6.

[13] Leung CK, Chiu V, Weinreb RN, et al. Evaluation of retinal nerve fiber layer progression in glaucoma: a comparison between spectral-domain and time-domain optical coherence tomography. Ophthalmology 2011; 118: 1558-62.

[14] Knight OJ, Girkin CA, Budenz DL, et al. Effect of race, age, and axial length on optic nerve head parameters and retinal nerve fiber layer thickness measured by Cirrus HD-OCT. Arch Ophthalmol 2012; 130: 312-8.

[15] Girkin CA, McGwin G Jr, Sinai MJ, et al. Variation in optic nerve and macular structure with age and race with spectral-domain optical coherence tomography. Ophthalmology 2011; 118: 2403-8.

[16] Tariq YM, Li H, Burlutsky G, Michell P. Retinal nerve fiber layer and optic disc measurements by spectral domain OCT: normative 
values and associations in young adults. Eye (Lond). 2012; 26(12): 1563-70.

[17] Kremmer S, Zadow T, Steuhl KP, et al. Scanning laser polarimetry in myopic and hyperopic subjects. Graefes Arch Clin Exp Ophthalmol 2004; 242: 489-94.

[18] Leung CK, Yu M, Weinreb RN, et al. Retinal nerve fiber layer imaging with spectral-domain optical coherence tomography: interpreting the RNFL maps in healthy myopic eyes. Invest Ophthalmol Vis Sci 2012; 53: 7194-200.

[19] O'Leary N, Artes PH, Hutchison DM, et al. Rates of retinal nerve fiber layer thickness change in glaucoma patients and control subjects. Eye 2012; 26: 1554-62.
[20] Zhu H, Crabb D, Schlottmann P, et al. Aligning scan acquisition circles in optical coherence tomography images of the retinal nerve fiber layer. IEEE Trans Med Imaging 2011; 30: 1228-38.

[21] Naghizadeh F, Garas A, Vargha P, et al. Comparison of long-term variability of retinal nerve fiber layer measurements made with the RTVue OCT and scanning laser polarimetry. Eur J Ophthalmol. 2012; 31:0. doi: 10.5301/ejo.5000178. [Epub ahead of print].

[22] Kremmer S, Keienburg M, Anastassiou G, et al. Scanning laser topography and scanning laser polarimetry: comparing both imaging methods at same distances from the optic nerve head. Open Ophthalmol J 2012; 6: 6-16.

This is an open access article licensed under the terms of the Creative Commons Attribution Non-Commercial License (http://creativecommons.org/licenses/by-nc/3.0/) which permits unrestricted, non-commercial use, distribution and reproduction in any medium, provided the work is properly cited. 